\title{
EDITORIAL
}

\section{Apical ballooning of the left ventricle: a distinct entity?}

\author{
Y Abe, M Kondo
}

Heart 2003;89:974-976

Primary transient left ventricular apical ballooning is a distinct entity that has been diagnosed worldwide. Although neurogenic stunned myocardium has often been suggested as the cause, further investigation is recommended

See end of article for authors' affiliations

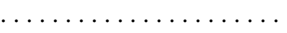

Correspondence to: Makoto Kondo, MD,

Division of Cardiology,

Shimada Municipal

Hospital, 1200-5 Noda

Shimada City, Shizuoka

427-8502 Japan

kondo-m@gb3.so-net.ne.jp
T ransient left ventricular apical ballooning is a syndrome presenting a unique morphologic feature of the left ventricle, characterised as a reversible balloon-like left ventricular wall motion abnormality at the apex with hypercontraction of the basal segment (fig 1). This entity was introduced as "tako-tsubo-like left ventricular dysfunction" in 1990 by Satoh and colleagues, and several reports have followed in Japan. ${ }^{1-5}$ "Tako-tsubo" is a fishing pot with a round bottom and narrow neck, which is used for trapping octopuses in Japan. ${ }^{4}$ However, establishment of its name, aetiology, criteria, management, and outcome remain challenging tasks.

Recently, we reported the clinical features in this syndrome. ${ }^{5}$ The median age of our patients was 74 years, and $82 \%$ of patients were female, consistent with previous studies. ${ }^{2-4} 6$ Almost all patients had physical or emotional stress as triggering factors with minimum evidence of coronary risk factors. On ECG, ST-T segment elevation or inverted $\mathrm{T}$ waves with a prolonged QTc interval were observed in several leads during the acute phase and returned to normal during chronic phase. ${ }^{5}$ Because these characteristics are similar to acute myocardial infarction (AMI), it is important to distinguish it from AMI to avoid unnecessary emergency coronary angiography or intravenous thrombolytic treatment. A limited release of cardiac enzyme compared with the large area of left ventricular dysfunction may be a reference feature to help distinguish it from AMI. ${ }^{2-5}$

\section{STUNNED MYOCARDIUM?}

On the other hand, prolonged post-ischaemic ventricular dysfunction has been reported as ischaemic stunned myocardium. ${ }^{7}$ All reports about this syndrome demonstrated that no significant stenosis occurred in the epicardial coronary artery. ${ }^{1-5}$ A focal coronary vasospasm with ST segment elevation was induced in a limited number of patients and the discrepancy between the hypokinetic area and coronary artery territories, which induced coronary vasospasm, were observed. ${ }^{235}$ Although Kuris and colleagues suggested that simultaneous multivessel coronary spasm at the epicardial coronary artery or microvascular level may contribute to this syndrome, this finding was considered speculation. ${ }^{4}$ No patients revealed angiographical spasm or slow flow during ST segment elevation at the acute phase in our report. ${ }^{5}$ Moreover, we demonstrated that microvascular function estimated by Doppler guidewire was almost normal, and contrast enhanced myocardium at the apex of the ventricle was observed by myocardial contrast echocardiography. ${ }^{5}$ Thus, we believe that damage
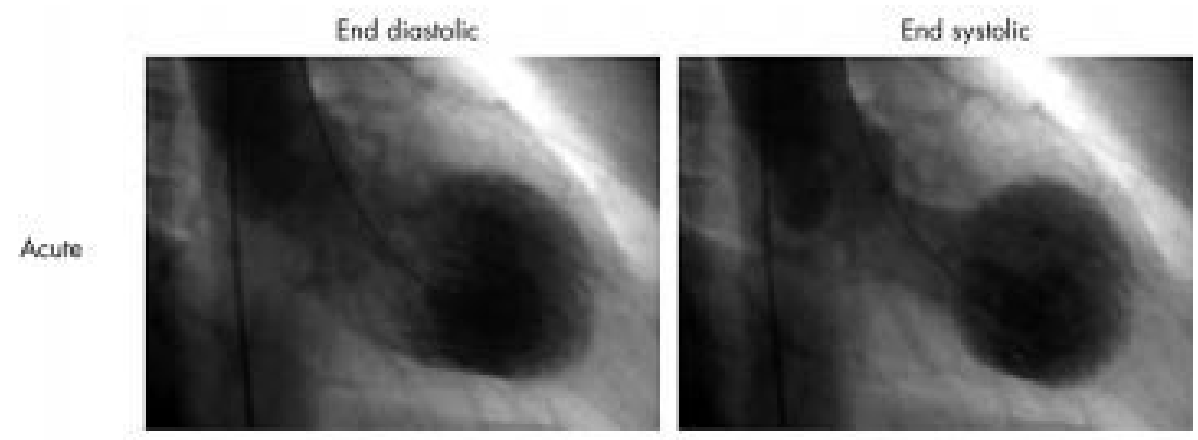

Figure 1 Left ventriculogram during the acute and chronic phase.

Reversible balloon-like asynergy at the apex with hypercontraction of the basal segment of the ventricle was observed during the acute phase and disappeared during the chronic phase. The interval between left ventriculograms from acute to chronic phase was 51 days.

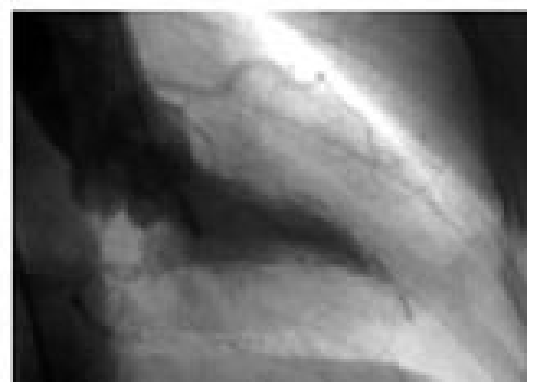


to the coronary circulation did not contribute to the aetiology.

We reported that technetium-99m tetrofosmin myocardial imaging revealed decreased uptake at the apex of the left ventricle in almost all patients during the acute phase that later returned to uniform. ${ }^{5}$ We supposed that the scintigraphic abnormality represented the abnormality of mitochondria from the mechanism of uptake of the tracer, because our patients had no significant coronary circulation abnormalities.

\section{PATHOLOGICAL FINDINGS}

Pathological findings of this syndrome obtained from endomyocardial biopsy have been reported. ${ }^{25}$ Kawai and colleagues demonstrated focal myocyte injury, myocardial depletion with cell infiltrates including polymorphonuclear leucocytes, or fibrosis. ${ }^{2}$ Kurise and colleagues revealed focal myocytolysis, mild mononuclear cell infiltration, and slight increase of loose connective tissue. ${ }^{4}$ We demonstrated that interstitial fibrosis was a common finding and no significant inflammatory infiltration or necrosis of adjacent myocytes was seen during the acute phase. ${ }^{5}$ Thus, there was no evidence of acute myocarditis from these findings.

Previous reports demonstrated that a small number of patients with this syndrome were found to have arrhythmias (atrioventricular block, sinus bradycardia, paroxysmal atrial fibrillation, ventricular tachycardia, and ventricular fibrillation), pulmonary oedema, and cardiogenic shock as complications. ${ }^{2-4}$ Moreover, Tshuchihashi and colleagues commented that some patients required intravenous dopamine or dobutamine infusion and mechanical supports, including a percutaneous cardiopulmonary support system. ${ }^{3}$ In addition, it is important to clarify that there is recurrence of this syndrome for optimal management. Tsuchihashi and colleagues reported that two of 72 patients $(2.7 \%)$ exhibited recurrence caused by emotional stress, and one patient died suddenly. ${ }^{3}$ Although almost all patients with this syndrome were reported to have a favourable prognosis, careful clinical observation for the critical complications or recurrences is recommended.

In this issue of Heart, Desmet and colleagues report apical ballooning of the left ventricle in 13 white patients. ${ }^{6}$ Their data provide further evidence that not only Japanese but also patients of European descent fall victim to this syndrome.

A very interesting aspect of this observation is the pathological findings at necropsy of one patient who died of multiple organ failure. They reported that the patient had no macroscopic signs of recent myocardial infarction or scars. Moreover, microscopic examination revealed normal myocardial tissue, except for some fatty infiltration. This observation signifies that acute myocarditis does not contribute to the aetiology of this syndrome. Moreover, this is the first report to our knowledge of necropsy information on this syndrome.

\section{A DISTINCT ENTITY?}

Based on previous reports, this syndrome is characterised as: (1) reversible balloon-like left ventricular wall motion abnormality at the apex with hypercontraction of the basal segment; (2) ST-T segment abnormalities on ECGs mimicking AMI; (3) minimal evidence of coronary circulation abnormality; (4) induced by physical and emotional stress in most cases; (5) elderly females were common; and (6) having a favourable prognosis. ${ }^{1-5}$ Several entities with reversible left ventricular wall motion abnormalities including ischaemic myocardial stunning, subarachnoid haemorrhage, pheochromocytoma crisis, acute myocarditis, and tachycardia induced cardiomyopathy have been reported..$^{711}$ Therefore, we categorised this syndrome as primary (idiopathic) transient left ventricular apical ballooning that is distinct from the above entities, and transient left ventricular apical ballooning secondary to the above entities. We propose the diagnostic criteria of this syndrome (table 1). Furthermore, we consider that the name
Table 1 Diagnostic criteria for primary transient left ventricular apical ballooning

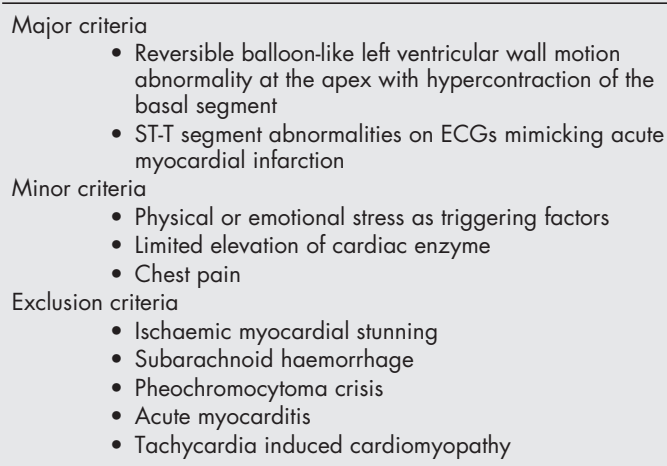

"transient left ventricular apical ballooning" is suitable for this syndrome. At the present time, this syndrome is known only by its morphological feature and the name should be more widely recognised outside Japan. Recently Tawarahara and colleagues reported a variant type of reversible severe left ventricular wall motion abnormality of the basal segment with hypercontraction at the apex. ${ }^{12}$ Therefore, the name may need to be re-evaluated in the future.

\section{NEUROGENIC STUNNED MYOCARDIUM AS SUGGESTED AETIOLOGY}

Almost all patients with this syndrome experienced triggering factors, including emotional or physical stress. ${ }^{35}$ Therefore, the participation by catecholamines is suggested. Recently, Ueyama and colleagues reported an experimental study in which left ventriculography of adult female Wistar rats exposed to immobilisation as an animal model of emotional stress showed that transient left ventricular apical ballooning was induced, which was normalised by pretreatment with $\alpha \beta$ adrenoceptor blockade. ${ }^{13}$ They also investigated the rapid activation of p44/p42 MAP kinase followed by a transient upregulation of immediate early genes in the rat heart, which was mediated by activation of adrenoceptors without the involvement of ischaemia.

In conclusion, the number of cases investigated in previous studies is relatively small and the aetiology remains unknown. The initiation of a large scale registry for suspected cases of this syndrome is highly desirable. It should be expanded to pathological assessment, molecular analysis, and experimental study. Moreover, definitions including primary and secondary causes appear crucial to understanding the aetiology.

\section{Authors' affiliations}

Y Abe, M Kondo, Division of Cardiology, Shimada Municipal Hospital, Shimada City, Shizuoka, Japan

\section{REFERENCES}

1 Satoh $\mathbf{H}$, Tateishi $\mathrm{H}$, Uchida $\mathrm{T}$, et al. Takotsubo-type cardiomyopathy due to multivessel spasm. In: Kodama K, Haze K, Hon M, eds. Clinical aspect of myocardial injury: from ischemia to heart failure [in Japanese] Tokyo: Kagakuhyouronsya Co, 1990;56-64.

2 Kawai S, Suzuki H, Yamaguchi H, et al. Ampulla cardiomyopathy ('Takotusbo' cardiomyopathy) - reversible left ventricular dysfunction with ST segment elevation. Jpn Circ J 2000;64:156-9.

3 Tsuchihashi K, Ueshima K, Uchida T, et al. Transient left ventricular apical ballooning without coronary artery stenosis: a novel heart syndrome mimicking acute myocardial infarction. J Am Coll Cardiol 2001;38:11-18.

4 Kurisu S, Sato H, Kawagoe T, et al. Tako-tsubo-like left ventricular dysfunction with ST-segment elevation: a novel cardiac syndrome mimicking acute myocardial infarction. Am Heart J 2002;143:448-55. 
5 Abe $Y$, Kondo M, Matsuoka R, et al. Assessment of clinical features in transient left ventricular apical ballooning. J Am Coll Cardiol 2003;41:737-42.

6 Desmet WJR, Adriaenssens BFM, Dens JAY. Apical ballooning of the left ventricle: first series in white patients. Heart 2003;89:1027-31.

7 Braunwald E, Kloner RA. The stunned myocardium: prolonged postischemic ventricular dysfunction. Circulation 1982;66:1146-9. 8 Kono T, Morita H, Kuroiwa T, et al. Left ventricular wall motion abnormalities in patients with subarachnoid hemorrhage: neurogenic stunned myocardium. J Am Coll Cardiol 1994;24:636-40.

9 Shaw TRD, Bafferty P, Tait GW. Transient shock and myocardial impairment caused by pheochromocytoma crisis. Br Heart J 1987;57:194-8
10 Miklozek CL, Crumpacker CS, Royal HD, et al. Myocarditis presenting as acute myocardial infarction. Am Heart J 1988;115:768-76.

11 Shinbane JS, Wood MA, Jensen DM, et al. Tachycardia-induced cardiomyopathy: a review of animal models and clinical studies. J Am Coll Cardiol 1997;29:709-15

12 Tawarahara K, Matoh F, Odagiri K, et al. Nuclear cardiac imaging in "Tsubo-like (Pod-like)" transient left ventricular dysfunction. Circ J 2001;65(suppl 1-A):420.

13 Ueyama $T$ Kasamatsu $\mathrm{K}$, Hano $T$, et al Emotional stress induces transient left ventricular hypocontraction in the rat via activation of cardiac adrenoceptors. Circ J 2002;66:712-13

\section{IMAGES IN CARDIOLOGY}

\section{Congenitally corrected transposition of the great arteries ( $\mathrm{L}$ transposition)}

A

48 year old woman under went diagnostic coronary angiography for atypical chest pains. During catheterisation the aorta was seen to be in an abnormal anterior position and subsequent cannulation of the coronary circulation proved extremely difficult requiring multiple catheters. Eventually a single coronary artery was cannulated, which was normal.

Subsequent transthoracic and transoesophageal echocardiography was arranged which revealed that the morphological left ventricle with the mitral valve lay on the right side in continuation with the venous right atrium and was connected to the pulmonary artery. The morphological right ventricle with the tricuspid valve lay on the left in continuation with the arterial left atrium and was connected to the aorta. The patient therefore had congenitally corrected transposition of the great arteries.

Echo clues to its diagnosis are: (1) the triscupid valve (TV) always lies closer to the apex when compared to the mitral valve (MV) and this will be apparent on the left side of the heart (upper and lower panels, middle column); (2) a moderator band and thicker trabeculation may be seen in the morphological right ventricle again on the left side of the heart; (3) a five chamber view will not be possible on the left side, the aorta can be imaged from the right side on occasions; (4) left ventricle/mitral papillary muscles always insert into the free walls, and right ventricle/tricuspid valves insert into one free wall and the septum.

Our patient did not have any complications or evidence of coronary artery
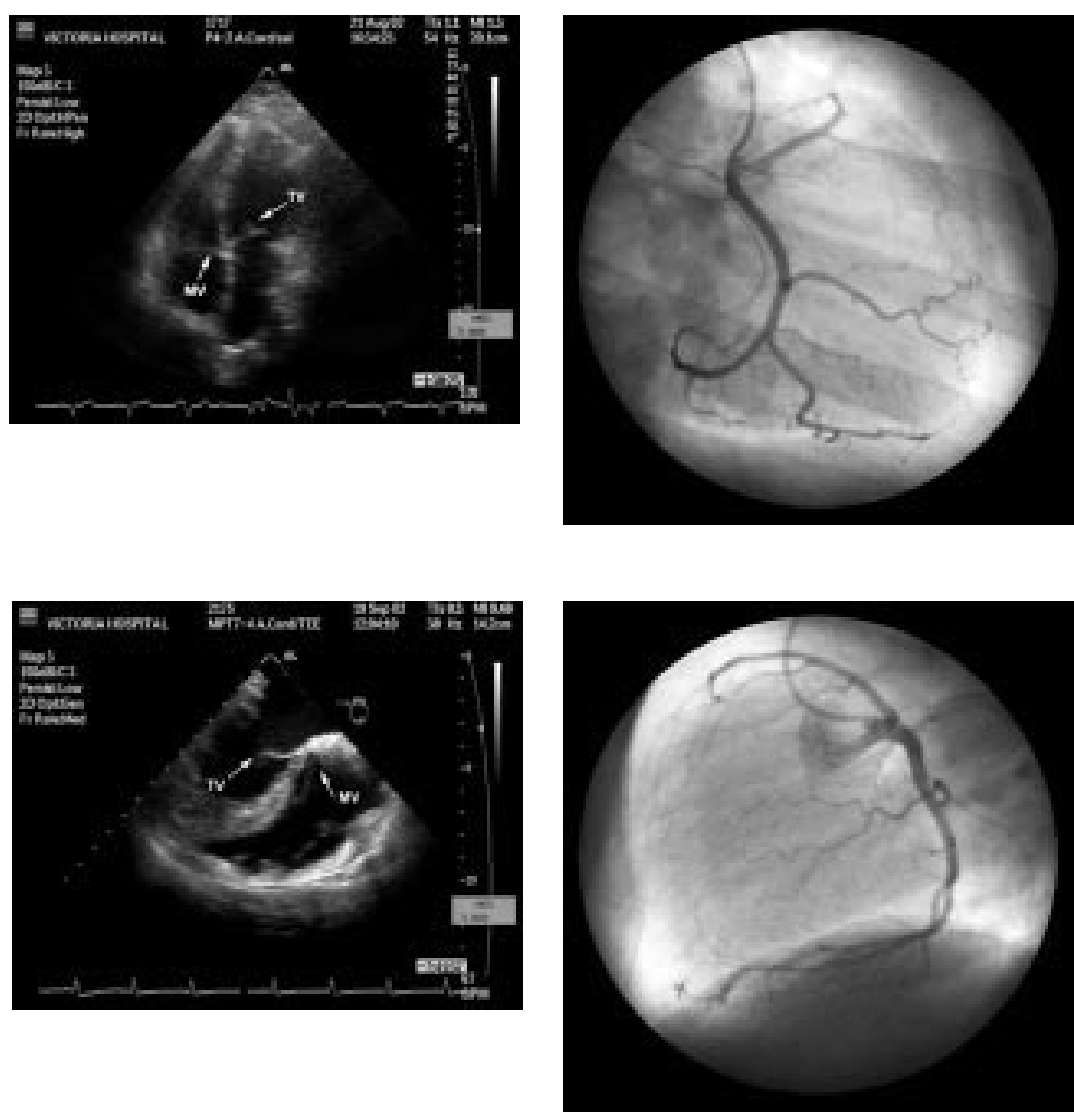

disease. Coronary angiography showed a single coronary artery origin, giving rise to a left anterior descending system and an equally dominant circumflex, with no right coronary artery (upper and lower panels, right column).

This is only the second known case of a congenitally corrected transposition of the great arteries ( $\mathrm{L}$ transposition) associated with a single coronary artery.

\section{S M Deepak \\ M Royle \\ G K Goode}

drdeepaksm@yahoo.co.uk 